\title{
Solutions of Modular Bootstrap Constraints from Quantum Codes
}

\author{
Anatoly Dymarsky $\oplus^{1,2}$ and Alfred Shapere $\oplus^{1}$ \\ ${ }^{1}$ Department of Physics and Astronomy, University of Kentucky, Lexington, Kentucky 40506, USA \\ ${ }^{2}$ Skolkovo Institute of Science and Technology, Skolkovo Innovation Center, Moscow 143026, Russia
}

(Received 14 November 2020; revised 5 January 2021; accepted 15 March 2021; published 21 April 2021)

\begin{abstract}
Modular invariance imposes rigid constraints on the partition functions of two-dimensional conformal field theories (CFTs). Many fundamental results follow strictly from modular invariance and unitarity, giving rise to the numerical modular bootstrap program. Here we report on a way to relate a particular family of quantum error correcting codes to a family of "code CFTs," which forms a subset of the space of Narain CFTs. This correspondence reduces modular invariance of the 2D CFT partition function to a few simple algebraic relations obeyed by a multivariate polynomial characterizing the corresponding code. Using this correspondence, we construct many explicit examples of physically distinct isospectral theories, as well as many examples of nonholomorphic functions, which satisfy all the basic properties of a 2D CFT partition function, yet are not associated with any known CFT.
\end{abstract}

DOI: $10.1103 /$ PhysRevLett.126.161602

Two-dimensional conformal field theories (CFTs) enjoy an exceptionally wide range of applications, from condensed matter physics to string theory and quantum gravity. Characterizing the space of all CFTs is one of the central tasks of the conformal modular bootstrap program [1-17], which aims to deduce universal properties of $2 \mathrm{D}$ theories, as well as details of specific models, from the modular invariance and non-negativity of their partition functions on the torus. In a nutshell, the modular bootstrap conditions form a proper subset of the conditions for conformal invariance and unitarity, and hence a solution to the bootstrap equations does not necessarily imply the existence of an actual theory. Nevertheless, it has been observed numerous times, including in the context of the conformal bootstrap in $d>2$, that a robust solution of the bootstrap constraints, e.g., a "kink" in the exclusion plot, reflects the presence of an actual theory. This picture is consistent with another observation that, with the exception of a limited family of examples related to chiral models [18] and a class of candidate partition functions for rational CFTs with two characters [19], all currently known nonchiral candidate partition functions-nonholomorphic modular-invariant functions $Z(\tau, \bar{\tau})$, which can be expanded in (Virasoro) characters with non-negative integral coefficients and with leading coefficient equal to one (reflecting the requirement of a unique CFT vacuum) - are partition functions of actual 2D theories. Furthermore, in practice, solutions of the

Published by the American Physical Society under the terms of the Creative Commons Attribution 4.0 International license. Further distribution of this work must maintain attribution to the author(s) and the published article's title, journal citation, and DOI. Funded by SCOAP ${ }^{3}$. modular bootstrap equations are typically associated with specific theories, which implicitly assumes that distinct CFTs must have different partition functions.

We show that this simple picture is not accurate, and the true situation is maximally complex: (i) there are many functions $Z(\tau, \bar{\tau})$ that are not partition functions of any (known) theory, and (ii) there are many examples of isospectral physically distinct theories, i.e., pairs, triplets, and even groups of 11 theories, which are all inequivalent but share the same partition function. Our results therefore expose the limitations of any approach, including the modular bootstrap program, which aims to characterize CFTs solely on the basis of their torus partition functions.

Our construction is very explicit and uses a map from a particular class of quantum error correcting codes to the space of 2D Narain CFTs that describe the compactification of free scalar fields on a multidimensional cube. The CFT partition function in this case is fully determined by the code, namely by the code's refined enumerator polynomial, introduced below. A direct search in the space of $n$-qubit codes with $n \leq 8$ readily reveals many dozens of distinct codes with the same enumerator, leading to many examples of physically distinct isospectral theories. There are also many polynomials obeying the standard symmetries and constraints of the enumerator polynomial, which are nevertheless not enumerator polynomials of any actual code. These "fake" polynomials provide thousands of examples of modular-invariant $Z(\tau, \bar{\tau})$, which are sums of $\mathrm{U}(1)^{n} \times \mathrm{U}(1)^{n}$ characters,

$$
Z(\tau, \bar{\tau})=\frac{1+\sum_{h, \bar{h}} C_{h, \bar{h}} q^{h} \bar{q}^{\bar{h}}}{|\eta(\tau)|^{2 n}}, \quad q=e^{2 \pi i \tau}
$$


with non-negative integral coefficients $C_{h, \bar{h}}$, and which are not partition functions of any known CFTs.

We start by reviewing graph codes, a particular family of real self-dual stabilizer codes [20,21]. A graph code is specified by a binary symmetric matrix $\mathcal{B}$-the adjacency matrix of an unoriented graph on $n$ nodes, $\mathcal{B}_{i i}=0$, $\mathcal{B}_{i j}=\mathcal{B}_{j i} \in\{0,1\}$. The adjacency matrix defines a set of $n$ generators

$$
g_{i}=\sigma_{x}^{i} \prod_{j=1}^{n}\left(\sigma_{z}^{j}\right)^{\mathcal{B}_{i j}}
$$

acting on the space of $n$ qubits. Because the matrix $\mathcal{B}$ is symmetric, the generators $g_{i}$ commute. Furthermore, they are nilpotent $g_{i}^{2}=I$ and they generate an Abelian stabilizer group $\mathcal{S}$ of rank $2^{n}$. There is a unique (up to scalar rescaling) state $\psi_{\mathcal{C}}$, which is invariant under the action of any element of the stabilizer group, $g_{i} \psi_{\mathcal{C}}=\psi_{\mathcal{C}}$. Known as the graph state [22-25], $\psi_{\mathcal{C}}$ can be written explicitly in terms of the "computational" up-down basis. Self-duality of the code implies that the Abelian stabilizer group possesses the maximal possible number of independent generators. The code is called real because all matrix elements of (2) are real.

The most general element of the stabilizer group is a product of generators

$$
g(\alpha)=\prod_{i=1}^{n} g_{i}^{\alpha_{i}}
$$

characterized by a binary vector $\vec{\alpha} \in \mathbb{Z}_{2}^{n}$. Up to a sign it can be written as a product of Pauli operators

$$
g(\alpha)=\epsilon \prod_{i=1}^{n}\left(\sigma_{x}^{i}\right)^{\alpha_{i}} \prod_{j=1}^{n}\left(\sigma_{z}^{j}\right)^{\beta_{j}}, \quad \epsilon= \pm 1,
$$

where the binary vector $\vec{\beta}:=\mathcal{B} \vec{\alpha} \bmod 2$. With this definition $w_{y}(\alpha)=\vec{\alpha} \cdot \vec{\beta}$ counts the number of Pauli matrices $\sigma_{y}$ in (4), while $w(\alpha)=\sum_{i} \alpha_{i}+\beta_{i}-w_{y}(\alpha)$ counts the total number of qubits on which $g(\alpha)$ acts nontrivially. A basic characteristic of a code is its enumerator polynomial, which counts the number of $g \in \mathcal{S}$ that act on a particular number of qubits. For our purposes, we consider the closely related refined enumerator polynomial, which keeps track of the total number of affected qubits, as well as the number of $\sigma_{y}$ 's,

$$
W(x, y, z)=\sum_{\vec{\alpha} \in \mathbb{Z}_{2}^{n}} x^{n-w(\alpha)} y^{w_{y}(\alpha)} z^{w(\alpha)-w_{y}(\alpha)} .
$$

When the code is real, there is always an even number of $\sigma_{y}$ in each $g(\alpha)$, and therefore $W$ is invariant under

$$
y \rightarrow-y .
$$

Furthermore, the refined enumerator polynomial of a selfdual code is invariant under the transformation

$x \rightarrow \frac{x+y+2 z}{2}, \quad y \rightarrow \frac{x+y-2 z}{2}, \quad z \rightarrow \frac{x-y}{2}$.

This symmetry follows from the MacWilliams identity [26-28].

In the context of quantum codes, it is natural to call codes equivalent if they are related by a permutation of qubits, which at the level of graphs is simply a relabeling of nodes. Two codes are also said to be equivalent if they are related by a local Clifford (LC) transformation, a unitary transformation $g_{i} \rightarrow U g_{i} U^{\dagger}$ acting on the individual qubits $U=u_{1} \otimes \ldots \otimes u_{n}$, which preserves the form of the stabilizer generators as tensor products of Pauli operators. If we restrict attention to real codes, the only allowed LC transformations are those generated by the Hadamard matrix, $u_{i}=H$, which simply exchanges $\sigma_{x}^{i} \leftrightarrow \sigma_{z}^{i}$. Following a LC transformation, the generators can be recombined to bring them again to graph form (2), so that the action of the LC group can be understood in terms of graph transformations. At the level of graphs, all code equivalence transformations among real codes generate an orbit in the space of graphs under the action of edge local complementation (ELC) [29]. The action of an ELC transformation on the graph adjacency matrix is as follows:

$$
\mathcal{B} \rightarrow[(D+I) \mathcal{B}+D][D \mathcal{B}+D+I]^{-1},
$$

where all operations, including matrix inversion, are understood mod 2, and $D$ is an arbitrary diagonal matrix.

Clearly, permutations of qubits and exchanges $\sigma_{x}^{i} \leftrightarrow \sigma_{z}^{i}$ do not change (5); therefore, two graph codes associated with graphs related by ELC will have the same refined enumerator polynomial.

In the context of quantum computation, real codes are not special and code equivalence is usually defined to include the full group of LC transformations. At the level of graphs, the full equivalence group gives rise to orbits under local complementation. A classification of orbits under local complementation for all graphs on $n \leq 12$ nodes has been performed in [30], where it was used to enumerate all equivalence classes of self-dual stabilizer codes for $n \leq 12$ qubits. The orbits of graphs under ELC are suborbits within the orbits of local complementation. To our knowledge, they have not been fully classified previously. We classify all ELC orbits for graphs on $n \leq 8$ nodes.

At this point, we would like to assign to each stabilizer group of the form (2) a Narain CFT, which describes $n$ free scalar fields compactified on an $n$-dimensional cube of "unit" size $2 \pi$ in the presence of quantized $B$-field flux. The Narain CFT can be defined in terms of an even self-dual Lorentzian lattice in $\mathbb{R}^{n, n}$. Starting from (2) or, equivalently, 
from the graph adjacency matrix $\mathcal{B}$, we define a lattice generator matrix

$$
\Lambda=\left(\begin{array}{c|c}
2 I & B \\
\hline 0 & I
\end{array}\right) / \sqrt{2},
$$

where $B_{i j}$ is an arbitrary antisymmetric matrix satisfying

$$
\mathcal{B}=B \bmod 2 .
$$

It is easy to check that (9) satisfies $\Lambda^{T} g \Lambda=g$, where the Lorentzian metric is

$$
g=\left(\begin{array}{l|l}
0 & I \\
\hline I & 0
\end{array}\right) .
$$

Different values of $B$ satisfying (10) lead to the same Lorentzian lattice, and the corresponding CFTs are related by $T$ duality. The relation between graph codes and Narain CFTs can be extended to all real self-dual codes [31]. We will call the corresponding theories code CFTs.

The partition function of a code CFT is fully specified by the underlying code itself or, more precisely, by its refined enumerator polynomial

$$
Z(\tau, \bar{\tau})=\frac{W_{\mathcal{C}}(b \bar{b}+c \bar{c}, b \bar{b}-c \bar{c}, a \bar{a})}{2^{n}|\eta(\tau)|^{2 n}},
$$

where $a=\theta_{2}(\tau), b=\theta_{3}(\tau)$, and $c=\theta_{4}(\tau)$. Using standard relations for the Jacobi theta functions, one can verify that (12) is invariant under $\tau \rightarrow \tau+1$ due to (6) and under $\tau \rightarrow-1 / \tau$ due to (7). This ensures modular invariance of the code CFT partition function. The refined enumerator polynomial satisfies other conditions, which follow from its definition: $W$ should be homogeneous, $W(1,0,0)=1$, and all coefficients of the polynomial $W(x, y, z)$ should be nonnegative integers. At the level of the partition function, this ensures $Z(\tau, \bar{\tau})$ is of the form (1) with non-negative integral $C_{h, \bar{h}}$.

The condition of invariance of the refined enumerator polynomial under (6) and (7) is easy to "solve" in full generality. A polynomial $W(x, y, z)$ invariant under both symmetries is an arbitrary polynomial in three generating polynomials,

$$
\begin{aligned}
& W_{1}=x+z, \quad W_{2}=x^{2}+y^{2}+2 z^{2}, \\
& W_{3}=x^{3}+3 x y^{2}+4 z^{3} .
\end{aligned}
$$

Imposing other conditions, $W(1,0,0)=1$ and non-negativity of integer coefficients, reduces the problem of finding such a $W$ to an exercise in discrete linear programming, which can be easily solved using computer algebra for small and moderate $n$. As is well known in the context of classical codes [28], there are many more polynomials satisfying the aforementioned conditions than there are actual codes. We call such polynomials that do not correspond to codes fake refined enumerator polynomials. Their number grows quickly with $n$. There are no fake polynomials for $n=1$ and $n=2$, there are six fake polynomials for $n=3$,

$$
\begin{gathered}
W=x^{3}+2 x^{2} z+3 x z^{2}+y^{2} z+z^{3}, \\
W=x^{3}+x^{2} z+3 x z^{2}+2 y^{2} z+z^{3}, \\
W=x^{3}+2 x^{2} z+x y^{2}+2 x z^{2}+2 z^{3}, \\
W=x^{3}+x y^{2}+2 x z^{2}+2 y^{2} z+2 z^{3}, \\
W=x^{3}+x^{2} z+2 x y^{2}+x z^{2}+3 z^{3}, \\
W=x^{3}+2 x y^{2}+x z^{2}+y^{2} z+3 z^{3},
\end{gathered}
$$

there are 11 for $n=4,128$ for $n=5,2835$ for $n=6,71$ 164 for $n=7,4012529$ for $n=8$, and so on. Each fake enumerator polynomial defines a candidate partition function via (12), which is modular invariant and non-negative, yet which is not the partition function of any known physical theory. The multitude of fake partition functions of small central charge that do not correspond to known CFTs is one of our main results.

$T$ duality is a symmetry of any Narain CFT; it leaves the physical theory intact, but can change the lattice generator matrix. In principle $T$ duality can map one code theory into another code theory. It is easy to see that the code equivalence transformations that respect the reality condition-permutations of qubits and exchanges of $\sigma_{x}^{i} \leftrightarrow \sigma_{z}^{i}$-become $T$-duality transformations, at the level of lattices and their associated Narain theories. The opposite is also true: any $T$-duality transformation that maps a code theory into another code theory is, at the level of codes, a code equivalence that involves qubit permutations and exchanges $\sigma_{x}^{i} \leftrightarrow \sigma_{z}^{i}$ [31]. We therefore arrive at a key observation: two code CFTs associated with graph codes are $T$-dual to each other (physically equivalent) if and only if the corresponding graphs are related by edge local complementation. Our classification of all ELC orbits for graphs on $n \leq 8$ nodes therefore provides an enumeration of all physically distinct code CFTs. By comparing refined enumerator polynomials associated with different ELC orbits, we find no degeneracies for $n \leq 6$ and one degeneracy for $n=7$. Namely, one refined enumerator

$$
\begin{aligned}
W= & x^{7}+x^{5} y^{2}+5 x^{4} y^{2} z+5 x^{2} y^{4} z+x^{5} z^{2} \\
& +12 x^{3} y^{2} z^{2}+9 x y^{4} z^{2}+4 x^{4} z^{3}+22 x^{2} y^{2} z^{3}+4 y^{4} z^{3} \\
& +5 x^{3} z^{4}+25 x y^{2} z^{4}+11 x^{2} z^{5}+11 y^{2} z^{5}+10 x z^{6}+2 z^{7}
\end{aligned}
$$



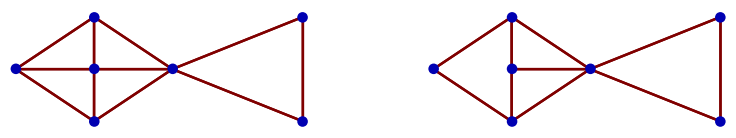

FIG. 1. "Fish" graphs-representatives of two ELC orbits of graphs, which at the level of graph codes share the same refined enumerator polynomial (20) and lead to a pair of isospectral nonchiral CFTs with $c=\bar{c}=7$.

is the same for two different classes of codes, giving rise to a pair of physically distinct isospectral CFTs. At the level of lattices, this corresponds to a pair of isospectral even selfdual Lorentzian lattices (also equipped with a Euclidean metric), analogous to Milnor's example of isospectral even self-dual lattices in $\mathbb{R}^{16}[32]$. At the level of graphs, the two ELC classes respectively include nine and ten different graphs (up to isomorphisms). We have chosen one graph from each class based on simplicity and aesthetics and have depicted them in Fig. 1.

For $n=8$, there are already 60 new pairs and 5 triplets of isospectral code theories, too many to list here. While we did not fully analyze ELC classes for higher $n$, we can utilize the analysis of [30] classifying orbits under local complementation. Since any ELC orbit is a subset within an orbit under local complementation, graphs belonging to different local complementation orbits correspond to physically different theories. Going through the values $n=9-11$ we find many examples of pairs, triples, and, in fact, $k$-tuples of isospectral code theories for every $k \leq 11$, confirming the expectation that the number of isospectral theories grows rapidly with $n$. These findings raise the question of identifying a mechanism that could explain the growing number of isospectral theories.

A natural generalization of our construction would be to consider the grand canonical partition function decorated by $\mathrm{U}(1)^{n} \times \mathrm{U}(1)^{n}$ charges, $Z(\tau, \bar{\tau}, \xi, \bar{\xi})$, where $\xi_{I}$ and $\bar{\xi}_{I}$ are the associated chemical potentials. The choice $\xi_{I}=\xi$ and $\bar{\xi}_{I}=\bar{\xi}$ for all $I=1, \ldots, n$ preserves permutation symmetry. With this choice, for code theories the grand canonical partition function is given in terms of the full code enumerator polynomial $W(t, x, y, z)$ by an expression generalizing (12)

$Z(\tau, \bar{\tau}, \xi, \bar{\xi})=\frac{W(b \bar{b}+c \bar{c}, a \bar{a}+d \bar{d}, b \bar{b}-c \bar{c}, a \bar{a}-d \bar{d})}{2^{n}|\eta(\tau)|^{2 n}}$,

where $a=\theta_{2}(\tau, \xi), \quad b=\theta_{3}(\tau, \xi), \quad c=\theta_{4}(\tau, \xi), \quad$ and $d=\theta_{1}(\tau, \xi)$. Just as one finds for $Z(\tau, \bar{\tau})$ in (12), modular covariance of $Z(\tau, \bar{\tau}, \xi, \bar{\xi})$ follows from the MacWilliams identity for $W(t, x, y, z)$. The introduction of nonzero $\xi, \bar{\xi}$ removes the degeneracy of $Z$ between the isospectral but $\operatorname{not} T$-dual $n=7$ theories associated with the graphs shown in Fig. 1. A detailed derivation of (21) together with a discussion of the $n=7$ case can be found in the Supplemental Material [33], which includes Ref. [34].

The construction relating quantum codes to rational CFTs outlined in this Letter is not unique. Other classes of codes can be naturally mapped to families of nonchiral rational theories [35]. As was shown in [36], conformal blocks of rational CFTs are wave functions of the dual Chern-Simons theory, which form a finite-dimensional Hilbert space. Provisionally, we identify this Hilbert space with the Hilbert space of qubits the quantum code describes. Exploring further the role of quantum codes in CFTs and in the holographic correspondence is an important program to which we hope to return in the future.

There is a simpler chiral version of our construction. Classical binary, doubly even self-dual codes are associated with even self-dual lattices, which can be used to define chiral CFTs. As is well known, starting with $n=24$ there exist fake enumerator polynomials and hence would-be holomorphic partition functions $Z(\tau)$, which are known to have no CFT counterpart [18]. Furthermore, starting in dimension $n=16$, there are isospectral self-dual lattices and hence isospectral physically inequivalent chiral theories [37-39]. The main difference of our work is that it applies to nonchiral theories, which are much less studied. Furthermore, our examples arise in large numbers at much smaller values of the central charge, with implications for the numerical conformal bootstrap.

As a final remark, we notice that the partition function (12) is a polynomial in code CFT partition functions associated with the polynomials (13) [31]. This observation suggests a simple way to construct many new modularinvariant $Z(\tau, \bar{\tau})$ by simply taking polynomials of any collection of CFT partition functions and making sure that the coefficients in the character expansion are non-negative integers and the vacuum character is unique. For homogeneous polynomials, each term has leading small- $q$ behavior $q^{-c / 12}$, so uniqueness of the vacuum is a constraint on the coefficients of the polynomial. The examples in this Letter show that this constraint has many nontrivial solutions. For an inhomogeneous polynomial in a single variable, uniqueness of the vacuum requires the coefficient of the leading term to be one, as in [40]. We expect that there are many more possibilities for inhomogenious polynomials in several variables, leading to a plethora of modular-invariant $Z(\tau, \bar{\tau})$ not associated with any CFT.

Conclusions.-In this Letter, we have reported on a way to associate graph codes, a family of quantum error correcting codes, to a specific class of Narain CFTs, whose elements we call code theories. Code CFTs describe free scalar fields compactified on a multidimensional cube in the presence of quantized $\mathcal{B}$-flux. This mapping between graph codes and CFTs provides a new way to study nonchiral theories. It allows us to construct many explicit examples of isospectral nonchiral theories, as well as many examples of would-be partition functions (1) which do not 
correspond to any known theories. These examples emphasize the fact that solutions of the modular bootstrap equations need not correspond to a unique CFT, nor indeed to any CFT at all.

Many technical details relevant to our presentation, including data necessary to construct pairs and triples of $n=8$ isospectral theories, can be found in the accompanying work [31].

We thank Petr Kravchuk and Xi Yin for discussions. A. D. is supported by the National Science Foundation under Grant No. PHY-2013812.

[1] S. Hellerman, A universal inequality for CFT and quantum gravity, J. High Energy Phys. 08 (2011) 130.

[2] S. Hellerman and C. Schmidt-Colinet, Bounds for state degeneracies in $2 \mathrm{~d}$ conformal field theory, J. High Energy Phys. 08 (2011) 127.

[3] C. A. Keller and H. Ooguri, Modular constraints on CalabiYau compactifications, Commun. Math. Phys. 324, 107 (2013).

[4] D. Friedan and C. A. Keller, Constraints on 2d CFT partition functions, J. High Energy Phys. 10 (2013) 180.

[5] J. D. Qualls and A. D. Shapere, Bounds on operator dimensions in $2 \mathrm{~d}$ conformal field theories, J. High Energy Phys. 05 (2014) 091.

[6] T. Hartman, C. A. Keller, and Bogdan Stoica, Universal spectrum of $2 \mathrm{~d}$ conformal field theory in the large c limit, J. High Energy Phys. 09 (2014) 118.

[7] J. D. Qualls, Universal bounds on operator dimensions in general 2d conformal field theories, arXiv:1508.00548.

[8] H. Kim, P. Kravchuk, and H. Ooguri, Reflections on conformal spectra, J. High Energy Phys. 04 (2016) 184.

[9] Y.-H. Lin, S.-H. Shao, Y. Wang, and X. Yin, $(2,2)$ superconformal bootstrap in two dimensions, J. High Energy Phys. 05 (2017) 112.

[10] T. Anous, R. Mahajan, and E. Shaghoulian, Parity and the modular bootstrap, SciPost Phys. 5, 022 (2018).

[11] S. Collier, Y.-H. Lin, and X. Yin, Modular bootstrap revisited, J. High Energy Phys. 09 (2018) 061.

[12] N. Afkhami-Jeddi, T. Hartman, and A. Tajdini, Fast conformal bootstrap and constraints on 3d gravity, J. High Energy Phys. 05 (2019) 087.

[13] M. Cho, S. Collier, and X. Yin, Genus two modular bootstrap, J. High Energy Phys. 04 (2019) 022.

[14] T. Hartman, D. Mazáč, and L. Rastelli, Sphere packing and quantum gravity, J. High Energy Phys. 12 (2019) 048.

[15] N. Afkhami-Jeddi, H. Cohn, T. Hartman, D. de Laat, and A. Tajdini, High-dimensional sphere packing and the modular bootstrap, J. High Energy Phys. 12 (2020) 066.

[16] N. Afkhami-Jeddi, H. Cohn, T. Hartman, and A. Tajdini, Free partition functions and an averaged holographic duality, J. High Energy Phys. 01 (2021) 130.

[17] F. Gliozzi, Modular bootstrap, elliptic points, and quantum gravity, Phys. Rev. Research 2, 013327 (2020).
[18] A. N. Schellekens, Meromorphic $c=24$ conformal field theories, Commun. Math. Phys. 153, 159 (1993).

[19] S. Mukhi, Classification of RCFT from holomorphic modular bootstrap: A status report, arXiv:1910.02973, and references therein.

[20] D. Schlingemann and R.F. Werner, Quantum errorcorrecting codes associated with graphs, Phys. Rev. A 65, 012308 (2001).

[21] D. Schlingemann, Stabilizer codes can be realized as graph codes, Quantum Inf. Comput. 2, 307 (2002).

[22] W. Dür, H. Aschauer, and H.-J. Briegel, Multiparticle Entanglement Purification for Graph States, Phys. Rev. Lett. 91, 107903 (2003).

[23] D. G. Glynn, T. Aaron Gulliver, J. G. Maks, and M. K. Gupta, The Geometry of Additive Quantum Codes (Springer, Berlin, 2006), p. 219.

[24] M. Van den Nest, J. Dehaene, and B. De Moor, Graphical description of the action of local clifford transformations on graph states, Phys. Rev. A 69, 022316 (2004).

[25] M. Hein, W. Dür, J. Eisert, R. Raussendorf, M. Nest, and H.-J. Briegel, Entanglement in graph states and its applications, arXiv:quant-ph/0602096.

[26] F. J. MacWilliams, A. M. Odlyzko, N. J. A. Sloane, and H. N. Ward, Self-dual codes over gf (4), J. Combinat. Theory Ser. A 25, 288 (1978).

[27] P. Shor and R. Laflamme, Quantum Analog of the MacWilliams Identities for Classical Coding Theory, Phys. Rev. Lett. 78, 1600 (1997).

[28] G. Nebe, E. M. Rains, and N. J. A. Sloane, Self-Dual Codes and Invariant Theory (Springer, New York, 2006), Vol. 17.

[29] M. Van den Nest and Bart De Moor, Edge-local equivalence of graphs, arXiv:math/0510246.

[30] L. E. Danielsen and M. G. Parker, On the classification of all self-dual additive codes over gf (4) of length up to 12 , J. Combinat. Theory Ser. A 113, 1351 (2006).

[31] A. Dymarsky and A. Shapere, Quantum stabilizer codes, lattices, and CFTs, J. High Energy Phys. 03 (2021) 160.

[32] J. H. Conway, The Sensual (Quadratic) Form (American Mathematical Society, Providence, 1997), Vol. 26.

[33] See Supplemental Material at http://link.aps.org/ supplemental/10.1103/PhysRevLett.126.161602 for a derivation of (21) and a discussion of its use in distinguishing isospectral theories. The Supplemental Material includes a reference to [34].

[34] P. Kraus and F. Larsen, Partition functions and elliptic genera from supergravity, J. High Energy Phys. 01 (2007) 002.

[35] A. Dymarsky and A. Sharon (to be published).

[36] E. Witten, Quantum field theory and the jones polynomial, Commun. Math. Phys. 121, 351 (1989).

[37] P. Ginsparg, On toroidal compactification of heterotic superstrings, Phys. Rev. D 35, 648 (1987).

[38] K. S. Narain, New heterotic string theories in uncompactified dimensions $<10$, Phys. Lett. 169B, 41 (1986).

[39] K. S. Narain, M. H. Sarmadi, and E. Witten, A note on toroidal compactification of heterotic string theory, Nucl. Phys. B279, 369 (1987).

[40] E. Witten, Three-dimensional gravity revisited, arXiv:0706.3359. 